\title{
Cognição, Emoção e Expertise Musical
}

\author{
Afonso Galvão ${ }^{1}$ \\ Universidade Católica de Brasília
}

\begin{abstract}
RESUMO - Este texto, sobre a relação entre cognição e música, objetiva lançar novas perspectivas para uma idéia há muito presente na pesquisa psicológica: de que a atividade musical tem importantes conseqüências para o desenvolvimento emocional e cognitivo da pessoa. Estas, no entanto, não são sempre positivas, já que processos de aprendizagem musical, da forma como tradicionalmente desenvolvidos, podem também levar à frustração e ao adoecimento tanto físico como psíquico. O texto analisa estes temas, tendo como foco a aprendizagem expert de instrumentos musicais da tradição clássica como uma aprendizagem permanente, que envolve o desenvolvimento e refinamento constante de habilidades metacognitivas e autoreguladoras, bem como uma capacidade para superar obstáculos emocionais oriundos do tipo de investimento necessário ao alcance e manutenção da expertise musical.
\end{abstract}

Palavras-chave: expertise; psicologia da música; desenvolvimento cognitivo; ansiedade; metacognição.

\section{Cognition, Emotion and Musical Expertise}

\begin{abstract}
This text, about the relationship between music and cognition, aims at launching new perspectives for an idea, which is quite old in psychological research, that musical activity has important consequences for emotional and cognitive human development. However, these are not always positive, once music learning processes, as traditionally developed, may also lead to frustration and illness, both physical as psychological. This text analyses these topics, focusing on expert learning of musical instruments of classical tradition as a form of continuous learning which involves the constant development and refining of metacognitive and self-regulatory abilities, as well as a capacity to overcome emotional obstacles derived from the type of investment necessary to achieve and maintain musical expertise.
\end{abstract}

Key words: expertise; musical psychology; cognitive development; anxiety; metacognition.

A música é uma das expressões fundamentais da cultura humana. Algumas imagens do Olodum, dos muitos grupos musicais do Recife que animam festas com estilos tão distintos, dos trios elétricos, das escolas de samba, do grupo de rock que anima bailes, são suficientes para dar conta dessa atividade na vida humana. Há a polêmica da sala de concertos lotada para a apresentação da nona de Beethoven, de que todo mundo gosta, ou a pouca platéia que assiste com um sorriso conflituoso à estréia da obra contemporânea que rompe com a idéia de nota musical, de que quase ninguém gosta. Música é assim: ama-se e odeia-se com argumentos acalorados e dicotômicos. A reação humana ao discurso musical raramente é de indiferença. Isso traduz a experiência musical como uma experiência emocional socialmente compartilhada em festas, funerais, salas de concerto, cinemas, carros e em muitos momentos da vida cotidiana.

A atividade musical, que é multifacetada em termos de estilo, também o é no que diz respeito aos modos de vivenciá-la. Alguém pode simplesmente sentar-se confortavelmente na sala de estar e colocar para tocar um CD com a versão de Carlos Kleiber da primeira de Mahler; ou pode dirigir o carro enquanto toca na própria mente uma representação acústica da obra que ouviu pela manhã. Além disso, pode-se cantarolar a parte melodiosa de

1 Endereço: Universidade Católica de Brasília, Campus II, Pós-Graduação e Pesquisa, SGAN 916, mod. B, Brasília, DF, Brasil 70790-160. E-mail: agalvao@pos.ucb.br uma peça qualquer, pode-se ouvir uma música a caminho do supermercado e emocionar-se pois era a que estava tocando quando da ruptura com a primeira namorada. Ao ouvir uma peça barroca inédita na experiência pessoal, o argumento caótico dos sons, de alguma forma se organiza. Devido a algum tipo de ordenação subjacente, fazemos sentido do que ouvimos e, dependendo do tipo de exposição já experimentada em relação àquele estilo, intuitivamente declaramos que isto que é ouvido agora deve ser Bach. Este "fazer sentido" de um discurso musical pode ser um conjunto de convenções arbitrárias, ou uma descrição da realidade, ou uma propriedade da mente. De alguma forma, face à experiência musical, tal como em muitas outras experiências cognitivas, o cérebro busca naturalmente por regularidades. Esta posição teórica tem sido defendida por estudiosos tão diferentes como David Hilbert e os primeiros psicólogos da Gestält.

Perceber o discurso dos sons musicais envolve perceber aspectos tais como simetria, repetição e imitação, algo comprovado por experimentos sobre padrões auditivos temporais (Garner, 1974; Jones, 1978). Tocar um instrumento musical é uma das mais complexas atividades humanas pelo tipo de demanda que faz ao sistema de conhecimento como um todo. Envolve uma interdependência de aspectos cognitivos, kinaestheticos e emocionais realizados por meio de uma coordenação entre os sistemas auditivos e visuais, que se articulam com o controle motor fino (Galvão \& Kemp, 1999; Pederiva, 2005). Para que a performance aconteça, há a necessidade de um plano cognitivo capaz de estabelecer uma intenção de comu- 
nicação de um discurso musical coerente estabelecido na interpretação, e de um plano físico, para levar a termo o que foi estabelecido no plano interpretativo.

Assim, a relação entre cognição e música pode ser explorada a partir de muitas facetas. Pode-se pensar o texto musical a partir dos limites impostos à sua percepção por fatores psicoacústicos, ou tratá-lo numa perspectiva gramatical, como análogo à linguagem, já que a música é hierarquicamente organizada e faz uso seletivo de sistemas neurais complexos que interrelacionam o ouvido, a voz e o cérebro. Um modo de caracterizar a performance instrumental é como um tipo de atividade de resolução de problemas que envolvem dimensões tais como objetivos, conteúdo, meio de aprendizagem, alocação de tempo, planejamento e avaliação de resultados. Isso interage com características específicas de um aprendiz que, incluem personalidade e estilo cognitivo, equilíbrio emocional, traço de ansiedade, entre outros. Tornar-se um expert instrumentista da tradição clássica é um processo longo, que pode levar até 20 anos (Hayes, 1981; Sosniak, 1985, 1990). Assim, para que a expertise seja atingida, aprendizes têm de ser capazes de superar sérias limitações de ordem física, emocional e cognitiva.

Este texto focaliza aspectos cognitivos e emocionais que emergem no contexto da performance musical expert. São muitas as dimensões cognitivas relacionadas à atividade musical. Aqui vamos explorar quatro delas - estudo deliberado, auto regulação, memória e ansiedade - como meio de provocar uma reflexão sobre o complexo uso do sistema cognitivo na performance musical.

\section{Estudo Deliberado}

O tornar-se músico implica estudo individual deliberado de longo prazo e em exposição a variadas formas de experiência musical (ouvir música, tocar em grupo). $\mathrm{O}$ estudo individual deliberado é tido como um dos fatores individuais mais importantes no desenvolvimento da expertise musical. Alguns autores (Ericsson, TeschRomer \& Krampe, 1993; Sloboda, Davidson, Howe \& Moore, 1996) consideram-no o "mais" importante. Isto representa um certo exagero. O desenvolvimento da expertise envolve muito estudo deliberado que interage com outros fatores tais como características cognitivas do aprendiz, estilo cognitivo, personalidade, condições ambientais, o que torna em especulação perigosamente reducionista singularizar um aspecto. De todo modo, o fator estudo deliberado está em acordo com uma das poucas leis gerais da psicologia cognitiva - a lei geral do estudo deliberado. Esta lei transforma em equação matemática o antigo ditado de que a prática traz a perfeição. Há evidência consistente para a idéia de que a expertise musical é alcançada somente depois de muitos anos de intenso estudo individual deliberado. Além disso, depois de atingida a expertise, músicos tem de continuar com estudo individual consistente se quiserem prolongar a carreira. Mas, o que significa este estudo deliberado? Que dimensões de aprendizagem envolve? Quais seus aspectos metacognitivos e auto-reguladores mais importantes?

Pesquisas de Ericsson e cols. (1993) e Sloboda e cols. (1996) foram conclusivas sobre a fundamental importância do estudo deliberado no desenvolvimento da experti$s e$. Diversos estudos (Bloom, 1985; Galvão, 2000; Krampe, 1994; Lacorte, 2006; Sosniak, 1990) sugerem pelo menos 16 anos de estudo deliberado para que alguém atinja a expertise instrumental. Outros trabalhos (Galvão, 2000; Krampe \& Ericsson, 1995; Manturzewska 1990) indicaram ser o estudo deliberado um hábito de vida inteira, cuja quantidade decresce consideravelmente após o período de conservatório. Músicos que se tornam experts iniciam estudos de instrumento muito cedo. O tempo de estudo, que começa com algo entre 20 minutos e uma hora por dia nos primeiros anos, aumenta para 25 horas semanais durante a adolescência e para até 35 durante o período de conservatório superior (Hallam, 1997).

Devido ao limitado número de investigações nessa área, não se sabe ainda como variáveis do tipo contexto educacional e tipo de instrumento influenciam a quantidade de estudo individual. A pesquisa de Jorgensen (1997), no entanto, coloca alguma luz nesta questão. Esta investigou o tempo de estudo individual de estudantes que tocam diferentes instrumentos em um conservatório da Noruega. Resultados sugerem que, como grupo, as cordas estudam por mais tempo e de modo mais uniforme, já que nenhum subgrupo pratica menos do que 26 horas por semana. Madeiras e metais mostraram uma variação de 10 horas na comparação entre músicos que estudam mais e menos.

No que diz respeito ao tempo de estudo individual, a literatura psicológica indica que estados de alta concentração mental só podem ser obtidos por cerca de 30 a 45 minutos. Indivíduos podem atingir um estado de fadiga mental após uma hora de trabalho mental intenso (Piperek, 1981). Em crianças, o limite temporal de atenção é ainda menor e tende a ser de um a um minuto e meio por ano de idade (Fontana, 1995). Em música, há a cultura de se pensar que quanto mais alguém pratica, melhor se torna. No entanto há limites físicos e psíquicos para a quantidade de prática. Embora haja especulação sobre pessoas que estudam até 70 horas por semana, não foi encontrado nem um único caso sistematicamente documentado de músico capaz de sustentar o estudo individual por mais do que 35 horas por semana no longo prazo. $\mathrm{Na}$ verdade, pesquisadores (Bastian, 1989; Ericsson \& cols., 1993; Hallam, 1995; Jorgensen, 1997) sugerem um máximo de quatro horas de estudo deliberado diário a ser sustentado no longo prazo.

Além da fadiga física, o estudo deliberado apresenta um outro problema, de ordem motivacional. A literatura (Ericsson \& cols., 1993; Galvão, 2000; Hallam, 1997; Sloboda \& cols., 1996; Sosniak, 1985, 1990) sugere ser o estudo deliberado uma atividade maçante e desprazerosa. Tocar um instrumento implica adiamento de gratificação. Pesquisa de minha autoria (Galvão, 2002) indica que isso não é tão simples como parece. Nos primeiros anos da aprendizagem de um instrumento para a expertise, aprendizes praticam sob pressão (dos pais, professores). No entanto, num segundo momento, geralmente na adolescência, há uma mudança gradual neste controle, com os estudantes assumindo progressivamente uma independência, em termos da responsabilidade pelo estudo individual. Na vida profissional, a motivação para a aprendizagem de uma peça vai ser fortemente influenciada pela fase de aprendizagem e pelo contexto. Por exemplo, nas primeiras sessões de estudo deliberado de uma obra complexa, há uma prática mais "clínica" (estudo de pequenas sessões, para ver afinação, execução de ritmo e notas separadamente, toca-se devagar um trecho, aumentando progressivamente a velocidade). Esta fase tende a ser consideravelmente mais maçante do que as etapas finais, quando alguém já está fazendo estudo de performance. Em músicos profissionais de orquestra sinfônica, o contexto parece exercer uma influência enorme no processo de aprendizagem. Eles freqüentemente têm de preparar um repertório que não escolheram em dois 
ou três dias, que é o tempo de ensaio para um concerto. Assim a dedicação ao estudo individual, neste caso vai depender de uma série de fatores tais como preferência pelo repertório, identificação com o maestro e momento profissional no contexto do grupo.

\section{Auto-Regulação}

A auto-regulação é um importante aspecto do processo de aprendizagem e uma função cognitiva que opera na base de sua ocorrência. Diz respeito aos mecanismos que as pessoas usam para controlar o seu próprio processo de aprendizagem. Implica estabelecer um objetivo ou norma de estudo e controlar o próprio progresso, utilizando estratégias tais como monitoração, elaboração e gerenciamento de esforço (Corno, 1989). Estas determinam decisões posteriores sobre continuar ou não alocando tempo para o estudo, bem como mudanças nas estratégias de aprendizagem (Thiede \& Dunlosky, 1999). Em geral, estudantes podem ser descritos como aprendizes autoregulados devido à ativa participação que têm em seu próprio processo de aprendizagem em termos de metacognição, comportamento e motivação. A auto-regulação tende a ser caracterizada em relação a estratégias, tais como, organização, transformação de informação, seqüenciamento, busca de informação, ensaio e estratégias mnemônicas. Estudo auto-regulado inclui ainda auto-reforçamento, busca de padrão, adiamento de gratificação, estabelecimento de objetivos, percepção de auto-eficácia, auto-instrução e auto-avaliação (Zimmerman, 1989).

A pesquisa sobre a auto-regulação no processo de aprendizagem de músicos tem focalizado três grandes áreas. Uma tenta descrever o comportamento auto-regulatório na prática de novatos (Weidenbach, 1996), estudantes avançados (Nielsen, 1999) e profissionais da tradição clássica (Miklaszewski, 1989) e da música popular (Lacorte, 2006). Outra área examina a auto-regulação como aspecto preditivo de sucesso na música instrumental (Weidenbach, 1996). Em outras palavras, trata-se de tentar verificar se o controle pessoal sobre o processo de aprendizagem é mais importante no alcance da expertise do que fatores como habilidade ou tempo de dedicação. Finalmente, há a preocupação de pesquisa sobre se a aprendizagem auto-regulada é treinável. Diversas investigações (Galvão, 2000; Kennel, 1989; Miklaszewski, 1989; Nielsen, 1999; Pogonowski, 1989) trouxeram evidência de boa qualidade de que comportamento de aprendizagem auto-regulado pode ser treinado e desenvolvido, estabelecendo formas sofisticadas de metacognição.

\section{Memorização}

Compreender a memória humana é uma das tarefas importantes da pesquisa psicológica e dos psicólogos cognitivos. Aqui a contribuição da Psicologia para a música tem sido considerável. Talvez devido ao esforço requerido, relativamente poucas performances, na tradição clássica, são empreendidas sem o auxílio da partitura, com a peça memorizada. Para que o processo de memorização ocorra, é necessário que haja diferentes dimensões de codificação (auditiva, visual, kinaesthetica) que acabam por tornar a aprendizagem em super aprendizagem e a performance mais consistente. Ao menos esta parece ser a impressão do público. Em investigação de Williamon (1999), o público que ouviu suítes de Bach para violoncelo solo com e sem memorização considerou execuções do primeiro tipo superiores. Isto parece concordar com os resultados da pesquisa de Davidson
(1993) de que tocar de cor aumenta a comunicabilidade entre músico e audiência, influenciando positivamente a percepção da expressão musical.

No que diz respeito a processos de memorização, nos últimos 100 anos, diversas investigações, a maioria experimental, focalizaram o modo como músicos memorizam, com resultados mais ou menos similares. Uma série de investigações desenvolvidas por Rubin-Rabson (1937, 1939, 1940, 1941) sobre processos de memorização de pianistas indicou estratégias que incluíam pré-estudo analítico (análise da estrutura do texto musical: estrutura harmônica, estilo, organização fraseológica, entre outros) anterior à prática física, distribuição de tempo de estudo, estudo de trechos curtos com mãos separadas e estudo mental. A importância do pré-estudo analítico foi apoiada por outras investigações (Galvão, 2000; Kopiez, 1991; Ross, 1964), como também a adoção de codificações múltiplas (Hallam, 1997; Lim \& Lipmann, 1991; Nuki, 1984). Estudos com maior preocupação ecológica (Miklaszewsky, 1995; Chaffin \& Imreh, 1994) indicaram que a memorização só se torna uma preocupação para o músico nos estágios mais avançados da aprendizagem. Enfatiza-se que um ponto comum destes estudos é a sugestão do uso de múltiplas formas de codificação (visual, auditiva e kinaesthetica) que, se trabalhadas eficientemente, acabam por integrar um esquema mental extremamente automatizado e que passa a demandar pouca atenção por parte do performer.

Nesse contexto, a análise musical ocupa um lugar particularmente importante. Pesquisas de McPherson (1996) e de minha própria autoria (Galvão, 2000) evidenciaram que, à medida que a expertise musical se desenvolve, músicos tendem a trabalhar utilizando estratégias cognitivamente mais elaboradas que levam em conta a natureza do material a ser memorizado, preferências individuais e possível nível de ansiedade performática. Em contraste, novatos tendem a confiar quase que exclusivamente, em processos diretos de automatização.

Deve ser enfatizado que a pesquisa sobre memória musical é ainda introdutória. Como argumenta Cohen (1996), quase nada se sabe sobre como a memória musical se desenvolve ou melhora e sobre quais processos envolvidos no ato de tocar mentalmente uma peça. Não sabemos se estes processos são similares ao "discurso interior" ou se diferentes vozes ou instrumentos variam em memorabilidade. Não sabemos também qual a analogia mais coerente para a música: linguagem, matemática, reconhecimento de faces, memória pictórica.

\section{Ansiedade}

A ansiedade é um tipo de emoção que interfere na realização de tarefas cognitivas. A maior parte da pesquisa sobre ansiedade tem considerado os seus aspectos debilitadores. Poucas dão ênfase à ansiedade como facilitadora da tarefa cognitiva. Para começar, é importante diferenciar entre traço e estado de ansiedade. $\mathrm{O}$ primeiro indica uma disposição geral para ser ansioso. Trata-se de estrutura individual. Estado de ansiedade representa uma situação mais transitória, a ser influenciada pelo tipo de contexto. No trabalho de Kemp (1997), o traço de ansiedade aparece como uma característica particularmente forte da personalidade de músicos que interage com aspectos tais como instabilidade emocional, tensão, suspeição, baixa auto-estima e apreensão. Se a ansiedade pode ou não ajudar na performance, isto depende de uma combinação de fatores.

De acordo com pesquisa de Hamann e Sobaje (1983), 
performances submetidas a altos níveis de estado de ansiedade foram consideradas superiores a performances em condições tranqüilas, com estudantes mais experientes exibindo uma performance ainda melhor. Depois foi demonstrado que o nível de proficiência também tinha um papel nisso, pois a alta ansiedade de traço foi considerada um fator facilitador na performance de estudantes com alto nível de domínio de tarefa e debilitador em caso de estudantes com baixa proficiência performática. Foi demonstrada, também, que uma correspondência entre o traço de ansiedade e um aumento no estado de ansiedade. Assim, pode ser concluído que o sucesso da performance num contexto estressante parece depender de um alto nível de domínio da tarefa que atua em combinação com o estado e traço de ansiedade. Lehrer (1987) considera ser possível transformar a ansiedade de debilitadora em facilitadora, mas para que isso ocorra é necessário o engajamento constante em atividade de prática de performance. Isto foi confirmado por músicos profissionais participantes da pesquisa de Galvão (2003).

\section{Conclusão}

Com base em toda a argumentação exposta neste texto, podemos então perguntar: que tipo de desenvolvimento a exposição à aprendizagem de um instrumento musical proporciona? Como mencionado, o estudo de um instrumento musical parece envolver, entre outras dimensões, um plano cognitivo e uma prática física para sustentá-lo e promovê-lo. Um processo de aprendizagem deste tipo é um típico exemplo da resolução de problemas em nível expert. Músicos aplicam diferentes estratégias para resolver problemas, criam representações elaboradas e desenvolvem suas habilidades por meio de estudo intenso e prolongado. Seus processos de memorização estão em acordo com a teoria dos níveis de processamento (Lockhart, 1972). Procedimentos de agrupamento estão na base da aprendizagem e são subjacentes a resultados de estudo, idéia desenvolvida por Rosenbloom e Newell (1986). Em outras palavras, no estudo deliberado de um instrumento musical, há a preocupação com a criação de uma regra procedimental capaz de permitir a execução de uma passagem do começo ao fim como uma unidade. Isto é atingido gradualmente a partir da resolução de problemas específicos que intermediam a unificação da passagem. O modelo ACT* (Anderson, 1987) parece plausível para explicar certos eventos típicos da aprendizagem de um instrumento. O ACT* (pronuncia-se "act star", que significa adaptive control of thought) é um modelo computacional do sistema cognitivo humano, que opera de forma serial (para uma análise detalhada deste modelo, ver Galvão, 2005). Propõe a existência de uma memória de produção que seria responsável pela automatização de movimentos. Conhecimento, que é inicialmente declarativo, torna-se procedimental por meio de um processo de procedimentalização que envolve uma redução em verbalização na medida em que a automatização aumenta. A memória procedimental açambarca estruturas de objetivos hierarquicamente organizados que por sua vez codificam planos de ação. Assim, o objetivo particular de tocar uma seqüência musical a torna uma fonte forte de ativação que vai de encontro às regras de produção que orientam a ação como um todo, prescindindo da ação dirigida. Deve-se, no entanto, salientar que, no momento, teorias de expertise, modelos computacionais e simbólico interacionistas de terceira geração oferecem respostas somente parciais para o fenômeno da expertise musical.

$\mathrm{Na}$ vida real, processos intuitivos relacionados à aprendizagem musical parecem maiores do que as explicações da teoria $\mathrm{ACT}^{*}$, por exemplo. Pesquisas sobre estudo deliberado e aprendizagem musical aqui mencionadas indicam que, na vida real, os modos de lidar com problemas musicais interagem com uma miríade de outros aspectos que não têm sido considerados de modo adequado por teorias cognitivas. Por exemplo, processos de aprendizagem de músicos parecem ser influenciados por respostas afetivas, isto é, por um tipo de relação afetiva com a peça, e com pessoas contextualmente importantes (maestro, professor) que podem exercer considerável influência no modo como uma peça é aprendida. Isto talvez exemplifique uma situação de aprendizagem resultante de uma interação entre emoção e cognição.

Em música, como em qualquer área de expertise, o estudo deliberado tem por objetivo atingir respostas automáticas, proficientes. Entretanto a pesquisa sobre aprendizagem musical (Galvão 2000; Hallam, 1997; Weidenbach, 1996) indica que o modo como músicos abordam problemas parece também automático. Isto é, músicos parecem possuir um repertório de resolução de problemas adaptável a diferentes problemas e uma capacidade para monitorar a adequação de respostas, modificando estratégias para atingir objetivos freqüentemente reavaliados enquanto a aprendizagem progride. Isto, no entanto, está longe de significar que músicos, mesmo profissionais, solucionam problemas de modo eficiente. Pelo contrário, de acordo com as pesquisas citadas, freqüentemente objetivos são apenas parcialmente atingidos. Uma razão para isto talvez sejam as limitações do repertório de resolução de problemas.

Por outro lado, deve-se considerar que, apesar das usualmente citadas similaridades, problemas musicais não são como problemas matemáticos. Em música, problemas são mais bem caracterizados como mal-definidos, o que permite, em certas situações, que muitas respostas possam ser consideradas corretas, a depender de perspectivas específicas. Assim, a escolha de estratégias de estudo parece influenciada por significados particulares que peças têm para músicos. Estes são até certo ponto cognitivos, mas parecem interagir com repostas intuitivas sobre que sabemos muito pouco. Isto implica investigar o conhecimento em um outro nível cuja natureza pode ser mais afetiva do que cognitiva.

Devido às suas demandas físicas, afetivas e cognitivas, a aprendizagem da música instrumental talvez seja uma das mais complexas aventuras humanas, o que certamente tem implicações para o desenvolvimento cognitivo, cujos limites ainda não somos capazes de apontar. Vimos ao longo deste texto que estudar um instrumento envolve uma experiência humana multifacetada e multidimensional. Compreender as diversas dimensões que compõem tal processo de aprendizagem pode fazer avançar não somente o nosso conhecimento sobre música, mas sobre a mente humana como um todo.

\section{Referências}

Anderson, J. (1987). Skill acquisition. Psychological Review, 94, 192-210.

Bastian, H. (1989). Leben fur Musik: Eine Biographie-Studie uber musikalische. (hoch)-Begabungen. Mainz: Schott.

Bloom, B. (1985). Developing talent in young people. New York: Ballentine.

Chaffin, R. \& Imreh, G. (1994). Memorising for piano performance: a case study of expert memory. Paper presented at the $3^{\text {th }}$ practical aspects of memory conference. University of Maryland, Washington. 
Cohen, G. (1996). Memory in the real world. London: Psychology Press.

Corno, L. (1989). Self-regulated learning: a volitional analysis. Em B. Zimmerman \& D. Schunk (Orgs.), Self-regulated learning and academic achievement: theory, research and practice (pp. 111-141). New York: Springer.

Davidson, J. (1993). Visual perception of performance manner in the movements of solo musicians. Psychology of Music Journal, 21, 103-113.

Ericsson, A., Tesch-Romer, C. \& Krampe, R. (1993). The role of deliberate practice in the acquisition of expert performance. Psychological Review, 100(3), 363-406.

Fontana, D. (1995). Psychology for teachers. London: BPS

Galvão, A. \& Kemp, A. (1999). Kinaesthesia and instrumental music instruction: some implications. Psychology of Music Journal, 27(2), 129-137.

Galvão, A. (2000). Practice in orchestral life: an exploratory study of string players learning processes. Tese de Doutorado, Reading University, Reading.

Galvão, A. (2002). Motivation to learn: the case of professional orchestra players. Em F. Mönks \& H. Wagner (Orgs), Proceedings of the $8^{\text {th }}$ Conference of The European Council for High Ability (pp. 62-70). Rodhes, Bonn: K. H. Bock.

Galvão, A. (2003). Anxiety and expert performance: a case study focusing on professional orchestra players. Trabalho apresentado na $24^{a}$ Conferência Internacional da Sociedade para a pesquisa sobre Stress e Ansiedade. Lisboa: Universidade Lusófona.

Galvão, A. (2005). O computador como metáfora da mente humana: alguns limites de aplicação. Em M. Freitas \& N. Venturinha (Orgs.), A expressão do indizível: estudos sobre filosofia e psicologia (pp. 315-334). Brasília: Universa.

Garner, W. (1974). The processing of information and structure. New York: Penguin.

Hallam, S. (1995). Professional musicians' orientations to practice: implications for teaching. British Journal of $\mathrm{Mu}$ sic Education, 12, 3-10.

Hallam, S. (1997). Approaches to instrumental music practice of experts and novices: implications for education. Em H. Jorgensen \& A. Lehmann (Orgs.), Does practice make perfect? current theory and research on instrumental music practice (pp. 3-27). Oslo: NMH - publikasjoner.

Hamman, D. \& Sobaje, M. (1983). Anxiety and the college musician: a study of performance conditions and subject variables. Psychology of Music, 11, 37-50.

Hayes, J. (1981). The complete problem-solver. Philadelphia: Franklin Institute Press.

Jones, R. (1978). Auditory patterns: studies in the perception of structure. Em P. Carterette \& L. Friedman (Orgs.), Handbook of perception (pp. 255-288). New York: Longman.

Jorgensen, H. (1997). Time for practising? higher level music students' use of time for instrumental music lessons. Em H. Jorgensen \& C. Lehman (Orgs.), Does practice make perfect? Current theory and research on instrumental music practice (pp. 42-58). Oslo: NMH.

Kemp, A. (1997). Individual differences in musical behaviour. Em D. Hargreaves \& A. North (Orgs.), The social psychology of music (pp. 25-45). Oxford: Oxford University Press.

Kennel, R. (1989). Musical thinking in the instrumental rehearsal. Em E. Boardman (Org.), Dimensions of musical thinking (pp. 212-226). Reston: Music Educators National Conference.

Kopiez, R. (1991). Structural aids to the cognitive practice of music: graphic or verbal analysis. Psychologica Belgica, $31(2), 163-171$.

Krampe, R. (1994). Maintaining excellence: cognitive-motor performance in in pianists differing in age and skill level. Berlin: Sigma.

Krampe, R. \& Ericsson, K. A. (1995). Deliberate practice and elite musical performance. Em J. Rink (Org.), The practice of performance (pp. 142-156). Cambridge: Cambridge University Press.

Lacorte, S. (2006). Aprendizagem do músico popular por meio da escuta atenta e intencional. Dissertação de Mestrado, Universidade Católica de Brasília, Brasília.
Lehrer, P. (1987). A review of the approaches to the management of tension and stage fright in musical performance. Journal of Research in Music Education, 35, 143-152.

Lim, S., \& Lippman, L. (1991). Mental practice and memorisation of piano music. The Journal of General Psychology, $118(1), 21-30$.

Lockhart, A. (1972). The nature of motor learning and skill. Em R. Singer (Org.), Readings in motor learning (pp. 931). Philadelphia: LEA and FEBIGER.

Manturzewska, M. (1990). A biographical study of the lifespan development of professional musicians. Psychology of Music, 18(2), 112-139.

McPherson, G. (1996). Five aspects of musical performance and their correlates. Bulletin of the Council for Research in Music Education, Special Issue, the $15^{\text {th }}$ Conference of the International Society for Music Education.

Miklaszewski, K. (1989). A case study of a pianist preparing a musical performance. Psychology of Music, 17, 95-109.

Miklaszewski, K. (1995). Individual differences in preparing a musical composition for public performance. Em M. Manturzewska, M. Miklaszewski \& A. Biatkowski (Orgs.), Psychology of music today (pp.16-29). Warsaw: Fryderyk Chopin Academy of Music.

Nielsen, G. (1999). Regulation of learning strategies during practice: a case study of a single church organ student preparing a particular work for a concert performance. Psychology of Music Journal, 27, 218-229.

Nuki, M. (1984). Memorisation of piano music. Psychologia, $27,157-163$

Pederiva, P. (2005). O corpo no processo ensino-aprendizagem de instrumentos musicais: percepção de professores. Dissertação de Mestrado, Universidade Católica de Brasília, Brasília.

Piperek, M. (1981). Stress and music: medical, psychological, and legal strain factors in a symphony orchestra musicians's profession. Vienna: WB.

Pogonowski, L. (1989). Metacognition: a dimension of musical thinking. Em E. Boardman (Org.), Dimensions of musical thinking (pp. 9-19). Reston: Music Educators National Conference.

Rosenbloom, P. \& Newell, A. (1986). The chunking of goal hierarchies: a generalised model of practice. Em R. Michalski \& J. Mitchell (Orgs.), Machine learning II: an artificial intelligence approach (pp. 312-342). Los Altos: Morgan Kaufmann.

Ross, E. (1964). Improving facilities in music memorisation. Journal of Research in Music Education, 12(4), 269-278.

Rubin-Rabson, G. (1937). The influence of analytic pre-study in memorising piano music. Archives of Psychology, 31, 153 .

Rubin-Rabson, G. (1939). Studies in the psychology of memorising piano music: I. A comparison of the unilateral and co-ordinated approach. Journal of Educational Psychology, $30(5), 321-345$.

Rubin-Rabson, G. (1940). Studies in the psychology of memorising piano music: II. A comparison of massed and distributed practice. Journal of Educational Psychology, 30, 270-284.

Rubin-Rabson, G. (1941). Studies in the psychology of memorising piano music: IV. The effect of incentive. Journal of Educational Psychology, 32, 45-54.

Sloboda, J., Davidson, J., Howe, M. \& Moore, D. (1996). The role of practice in the development of performing musicians. British Journal of Psychology, 87, 287-309.

Sosniak, L. (1985). Learning to be a concert pianist. Em B. S. Bloom (Org.), Developing talent in young people (pp. 1967). New York: Ballantine Books.

Sosniak, L. (1990). The tortoise, the hare, and the development of talent. Em M. Howe (Org.), Encouraging the development of exceptional skills and talents (pp. 149-164). Leicester: British Psychological Society.

Thiede, K. \& Dunlosky, J. (1999). Toward a general model of self-regulated study: an analysis of selection of items for study and self-paced study time. Journal of Experimental Psychology: Learning, Memory, and Cognition, 25(4), 1024-1037. 


\section{A. Galvão}

Weidenbach, V. (1996). The influence of self-regulation on instrumental music practice. Tese de Doutorado, University of Western Sydney, Nepean.

Williamon, A. (1999). The value of performing from memory. Psychology of Music Journal, 27, 84-95.

Zimmerman, B. (1989). A social cognitive view of self-regulated academic learning. Journal of Educational Psychology, 81(3), 329-339.

Recebido em 12.05.2005

Primeira decisão editorial em 15.03.2006

Versão final em 26.04.2006

Aceito em 25.05.2006 